Скопје, Македонија

\title{
SEQUENTIALLY CONVERGENT MAPPINGS AND COMMON FIXED POINTS OF MAPPINGS IN 2-BANACH SPACES
}

\author{
Martin Lukarevski ${ }^{1}$, Samoil Malčeski ${ }^{2}$
}

\begin{abstract}
In the past few years, the classical results about the theory of fixed point are transmitted in 2-Banach spaces, defined by A. White (see [3] and [8]). Several generalizations of Kannan, Chatterjea and Koparde-Waghmode theorems are given in [1], [4], [5] and [7]. In this paper, several generalizations of already known theorems about common fixed points of mappings in 2-Banach spaces, are proven, by using the sequentially convergent mappings.
\end{abstract}

\section{INTRODUCTION}

In 1968 White ([3]) introduces 2-Banach spaces. 2-Banach spaces are being studied by several authors, and certain results can be seen in [8]. Further, analogously as in normed space P. K. Hatikrishnan and K. T. Ravindran in [6] are introducing the term contraction mapping to 2-normed space as follows.

Definition 1 ([6]). Let $(L,\|\cdot, \cdot\|)$ be a real vector 2-normed space. The mapping $S: L \rightarrow L$ is contraction if there is $\lambda \in[0,1)$ such that

$$
\|S x-S y, z\| \leq \lambda\|x-y, z\| \text {, for all } x, y, z \in L .
$$

Regarding contraction mapping Hatikrishnan and Ravindran in [6] proved that contraction mapping has a unique fixed point in closed and restricted subset of 2-Banach space. Further, in [1], [4], [5] and [7] are proven more results related to fixed points of contraction mapping of 2-Banach spaces, and in [7] are proven several results for common fixed points of contraction mapping defined on the same 2-Banach space.

2010 Mathematics Subject Classification. 46J10, 46J15, 47H10

Key words and phrases. 2-normed spaces, 2-Banach spaces, fixed point, contraction mappings 
In our further considerations, we will give some generalizations of the above results for common fixed points of mapping defined on the same 2-Banach space. Thus, the mentioned generalizations we will do with the help of so-called sequentially convergent mappings which are defined as follows.

Definition 2. Let $(L,\|, \cdot\|)$ be a 2-normed space. A mapping $T: L \rightarrow L$ is said to be sequentially convergent if, for every sequence $\left\{y_{n}\right\}$, if $\left\{T y_{n}\right\}$ is convergent then $\left\{y_{n}\right\}$ also is convergent.

\section{COMMON FIXED POINTS OF MAPPING OF THE KANNAN TYPE}

Theorem 1. Let $(L,\|\cdot, \cdot\|)$ be a 2 - Banach space, $S_{1}, S_{2}: L \rightarrow L$ and mapping $T: L \rightarrow L$ is continuous, injection and sequentially convergent. If $\alpha>0, \gamma \geq 0$ are such that $2 \alpha+\gamma<1$ and

$$
\left\|T S_{1} x-T S_{2} y, z\right\| \leq \alpha\left(\left\|T x-T S_{1} x, z\right\|+\left\|T y-T S_{2} y, z\right\|\right)+\gamma\|T x-T y, z\|,
$$

for each $x, y, z \in L$, then $S_{1}$ and $S_{2}$ have a unique common fixed point $z \in L$. Proof. Let $x_{0}$ be an arbitrary point of $L$ and let the sequence $\left\{x_{n}\right\}$ be defined with $x_{2 n+1}=S_{1} x_{2 n}, x_{2 n+2}=S_{2} x_{2 n+1}$, for $n=0,1,2, \ldots$. If there is $n \geq 0$ such that $x_{n}=x_{n+1}=x_{n+2}$, then it is easy to prove that $u=x_{n}$ is a common fixed point for $S_{1}$ and $S_{2}$. Therefore, let's assume that there do not exist three different consecutive equal members of the sequence $\left\{x_{n}\right\}$. So, using inequalities (1), it is easy to prove that for each $n \geq 1$ and for each $z \in L$ the following holds true $\left\|T x_{2 n+1}-T x_{2 n}, z\right\| \leq \alpha\left(\left\|T x_{2 n+1}-T x_{2 n}, z\right\|+\left\|T x_{2 n}-T x_{2 n-1}, z\right\|\right)+\gamma\left\|T x_{2 n}-T x_{2 n-1}, z\right\|$ and

$$
\begin{gathered}
\left\|T x_{2 n-1}-T x_{2 n}, z\right\| \leq \alpha\left(\left\|T x_{2 n-2}-T x_{2 n-1}, z\right\|+\left\|T x_{2 n-1}-T x_{2 n}, z\right\|\right) \\
+\gamma\left\|T x_{2 n-2}-T x_{2 n-1}, z\right\|,
\end{gathered}
$$

from which it follows that

$$
\left\|T x_{n+1}-T x_{n}, z\right\| \leq \lambda\left\|T x_{n}-T x_{n-1}, z\right\|,
$$

for each $n=0,1,2, \ldots$, where $\lambda=\frac{\alpha+\gamma}{1-\alpha}<1$. Now from inequality (2) it follows that

$$
\left\|T x_{n+1}-T x_{n}, z\right\| \leq \lambda^{n}\left\|T x_{1}-T x_{0}, z\right\|,
$$


for each $z \in L$ and for each $n=0,1,2, \ldots$. But, then from inequality (3) follows that for each $m, n \in \mathbf{N}, n>m$ and for each $z \in L$ the following holds true

$$
\left\|T x_{n}-T x_{m}, z\right\| \leq \frac{\lambda^{m}}{1-\lambda}\left\|T x_{1}-T x_{0}, z\right\|,
$$

which means that the sequence $\left\{T x_{n}\right\}$ is Cauchy and because space $L$ is 2Banach we get that the sequence $\left\{T x_{n}\right\}$ is convergent. Further, the mapping $T: L \rightarrow L$ is sequentially convergent and because the sequence $\left\{T x_{n}\right\}$ is convergent, from definition 2 follows that the sequence $\left\{x_{n}\right\}$ is convergent, i.e. exists $u \in L$ such that $\lim _{n \rightarrow \infty} x_{n}=u$. Now from the continuity of $T$ follows that $\lim _{n \rightarrow \infty} T x_{n}=T u$. Then, for each $z \in L$ the following holds true

$$
\begin{aligned}
\left\|T u-T S_{1} u, z\right\| & \leq T u-T x_{2 n+2}, z\|+\| T x_{2 n+2}-T S_{1} u, z \| \\
= & \left\|T u-T x_{2 n+2}, z\right\|+\left\|T S_{2} x_{2 n+1}-T S_{1} u, z\right\| \\
& \leq\left\|T u-T x_{2 n+2}, z\right\|+\alpha\left(\left\|T u-T S_{1} u, z\right\|+\left\|T x_{2 n+1}-T S_{2} x_{2 n+1}, z\right\|\right) \\
& +\gamma\left\|T u-T x_{2 n+1}, z\right\| \\
& \leq\left\|T u-T x_{2 n+2}, z\right\|+\alpha\left(\left\|T u-T S_{1} u, z\right\|+\left\|T x_{2 n+1}-T x_{2 n+2}, z\right\|\right) \\
& +\gamma\left\|T u-T x_{2 n+1}, z\right\| .
\end{aligned}
$$

If in the last inequality we take that $n \rightarrow \infty$, for each $z \in L$ the following holds true

$$
\left\|T u-T S_{1} u, z\right\| \leq \alpha\left\|T u-T S_{1} u, z\right\|,
$$

and since $\alpha<1$, we conclude that $\left\|T S_{1} u-T u, z\right\|=0$, for each $z \in L$, i.e. $T S_{1} u=T u$. But, $T$ is injection, so $S_{1} u=u$, i.e. $u$ is fixed point on $S_{1}$. Analogously can be proved that $u$ is fixed point of $S_{2}$. Let $v \in L$ is another fixed point of $S_{2}$, i.e. $S_{2} v=v$. Then, for each $z \in L$ the following holds true

$$
\begin{aligned}
\|T u-T v, z\| & =\left\|T S_{1} u-T S_{2} v, z\right\| \\
& \leq \alpha\left(\left\|T u-T S_{2} v, z\right\|+\left\|T v-T S_{1} u, z\right\|\right)+\gamma\|T u-T v, z\| \\
& =(2 \alpha+\gamma)\|T u-T v, z\|,
\end{aligned}
$$

and as $2 \alpha+\beta<1$ we get that for each $z \in L$ the following holds true $\|T u-T v, z\|=0$, from which follows $T u=T v$. But, $T$ is injection, so $u=v$.

Corollary 1. Let $(L,\|\cdot, \cdot\|)$ be a 2 - Banach space, $S_{1}, S_{2}: L \rightarrow L$ and mapping $T: L \rightarrow L$ is continuous, injection and sequentially convergent. If $\alpha>0, \gamma \geq 0$ are such that $2 \alpha+\gamma<1$ and 


$$
\left\|T S_{1} x-T S_{2} y, z\right\| \leq \alpha \frac{\left\|T x-T S_{1} x, z\right\|^{2}+\left\|T y-T S_{2} y, z\right\|^{2}}{\left\|T x-T S_{1} x, z\right\|+\left\|T y-T S_{2} y, z\right\|}+\gamma\|T x-T y, z\|,
$$

for each $x, y, z \in L, z \neq 0$, then $S_{1}$ and $S_{2}$ have a unique common fixed point $z \in L$.

Proof. From inequality of condition follows inequality (1). Now the assertion follows from Theorem 1 .

Corollary 2. Let $(L,\|\cdot, \cdot\|)$ be a 2 - Banach space, $S_{1}, S_{2}: L \rightarrow L$ and mapping $T: L \rightarrow L$ is continuous, injection and sequentially convergent. If $0<\lambda<1$ and

$$
\left\|T S_{1} x-T S_{2} y, z\right\| \leq \lambda \cdot \sqrt[3]{\left\|T x-T S_{1} x, z\right\| \cdot\left\|T y-T S_{2} y, z\right\| \cdot\|T x-T y, z\|},
$$

for each $x, y, z \in L$, then $S_{1}$ and $S_{2}$ have a unique common fixed point $z \in L$.

Proof. From the inequality between the arithmetic and geometric mean follows that

$$
d\left(T S_{1} x, T S_{2} y\right) \leq \frac{\lambda}{3}\left(d\left(T x, T S_{1} x\right)+d\left(T y, T S_{2} y\right)+\beta d(T x, T y)\right) .
$$

Now the assertion follows from Theorem 1 for $\alpha=\gamma=\frac{\lambda}{3}$.

Corollary 3. Let $(L,\|\cdot\|$,$) be a 2$ - Banach space, $S_{1}^{p}, S_{2}^{q}: L \rightarrow L, p, q \in \mathbf{N}$ and mapping $T: L \rightarrow L$ is continuous, injection and sequentially convergent. If $\alpha>0, \gamma \geq 0$ are such that $2 \alpha+\gamma<1$ and

$$
\left\|T S_{1}^{p} x-T S_{2}^{q} y, z\right\| \leq \alpha\left(\left\|T x-T S_{1}^{p} x, z\right\|+\left\|T y-T S_{2}^{q} y, z\right\|\right)+\gamma\|T x-T y, z\|,
$$

for each $x, y, z \in L$. Then $S_{1}$ and $S_{2}$ have a unique common fixed point $u \in L$.

Proof. From Theorem 1 follows that mappings $S_{1}^{p}$ and $S_{2}^{q}$ have a unique common fixed point $u \in L$. That means $S_{1}^{p} u=u$, so $S_{1} u=S_{1}\left(S_{1}^{p} u\right)=S_{1}^{p}\left(S_{1} u\right)$, and $S_{1} u$ is fixed point of $S_{1}^{p}$. Analogously, we can prove that $S_{2} u$ is fixed point of $S_{2}^{q}$. But, from the proof of Theorem 1 follows that mappings $S_{2}^{q}$ and $S_{1}^{p}$ have unique fixed point, so $u=S_{2} u$ and $u=S_{1} u$. According to that, $u \in L$ is a unique common fixed point of $S_{1}$ and $S_{2}$. Clearly, if $v \in L$ is another unique common fixed point of $S_{1}$ and $S_{2}$, then it is a common fixed point of $S_{1}^{p}$ and $S_{2}^{q}$. But, $S_{1}^{p}$ and $S_{2}^{q}$ have a unique common fixed point, so $v=u$. 
Remark 1. Mapping $T: L \rightarrow L$ defined by $T x=x, x \in L$ is sequentially convergent. Therefore, if in theorem 1 and the corollaries 1,2 and 3 we take that $T x=x$ follows Theorem 4 and corollaries 6,7 and 8, [7].

\section{COMMON FIXED POINTS OF MAPPINGS OF CHATTERJEA TYPE}

Theorem 2. Let $(L,\|\cdot, \cdot\|)$ be a 2 - Banach space, $S_{1}, S_{2}: L \rightarrow L$ and mapping $T: L \rightarrow L$ is continuous, injection and sequentially convergent. If $\alpha>0, \gamma \geq 0$, are such that $2 \alpha+\gamma<1$ and

$$
\left\|T S_{1} x-T S_{2} y, z\right\| \leq \alpha\left(\left\|T x-T S_{2} y, z\right\|+\left\|T y-T S_{1} x, z\right\|\right)+\gamma\|T x-T y, z\|,
$$

for each $x, y, z \in L$, then $S_{1}$ and $S_{2}$ have a unique common fixed point $u \in L$.

Proof. Let $x_{0}$ is arbitrary point from $L$ and the sequence $\left\{x_{n}\right\}$ is defined with $x_{2 n+1}=S_{1} x_{2 n}, x_{2 n+2}=S_{2} x_{2 n+1}$, for $n=0,1,2, \ldots$. If there is $n \geq 0$ such that $x_{n}=x_{n+1}=x_{n+2}$, then $u=x_{n}$ is common fixed point of $S_{1}$ and $S_{2}$. Therefore, let's assume that there are three different consecutive equal members of the sequence $\left\{x_{n}\right\}$. Then, from nequality (4) follows that for every $z \in L$ and for every $n \geq 1$ the following holds true

$$
\begin{gathered}
\left\|T x_{2 n+1}-T x_{2 n}, z\right\| \leq \alpha\left(\left\|T x_{2 n-1}-T x_{2 n}, z\right\|+\left\|T x_{2 n}-T x_{2 n+1}, z\right\|\right) \\
+\gamma\left\|T x_{2 n}-T x_{2 n-1}, z\right\|,
\end{gathered}
$$

and

$$
\begin{gathered}
\left\|T x_{2 n-1}-T x_{2 n}, z\right\| \leq \alpha\left(\left\|T x_{2 n-2}-T x_{2 n-1}, z\right\|+\left\|T x_{2 n-1}-T x_{2 n}, z\right\|\right) \\
+\gamma\left\|T x_{2 n-2}-T x_{2 n-1}, z\right\|,
\end{gathered}
$$

so for each $z \in L$ and for each $n=0,1,2, \ldots$ the following holds true

$$
\left\|T x_{n+1}-T x_{n}, z\right\| \leq \lambda\left\|T x_{n}-T x_{n-1}, z\right\|,
$$

where $\lambda=\frac{\alpha+\gamma}{1-\alpha}<1$. Then, for each $z \in L$ and for each $n=0,1,2, \ldots$ the following holds true

$$
\left\|T x_{n+1}-T x_{n}, z\right\| \leq \lambda^{n}\left\|T x_{1}-T x_{0}, z\right\| .
$$

Furthermore, using the inequality (5), in the same way as in the proof of Theorem 1 can be proved that the sequence $\left\{T x_{n}\right\}$ is convergent, from where it follows that the sequence $\left\{x_{n}\right\}$ is convergent, i.e. there is $u \in L$ such that $\lim _{n \rightarrow \infty} x_{n}=u$ and $\lim _{n \rightarrow \infty} T x_{n}=T u$. We will prove that $u$ is a fixed point of $S_{1}$. For each $z \in L$ we have 


$$
\begin{aligned}
\left\|T u-T S_{1} u, z\right\| & \leq T u-T x_{2 n+2}, z\|+\| T x_{2 n+2}-T S_{1} u, z \| \\
= & \left\|T u-T x_{2 n+2}, z\right\|+\left\|T S_{2} x_{2 n+1}-T S_{1} u, z\right\| \\
\leq & \left\|T u-T x_{2 n+2}, z\right\|+\alpha\left(\left\|T x_{2 n+1}-T S_{1} u, z\right\|+\left\|T u-T S_{2} x_{2 n+1}, z\right\|\right) \\
& +\gamma\left\|T u-T x_{2 n+1}, z\right\| \\
\leq & \left\|T u-T x_{2 n+2}, z\right\|+\alpha\left(\left\|T x_{2 n+1}-T S_{1} u, z\right\|+\left\|T u-T x_{2 n+2}, z\right\|\right) \\
& +\gamma\left\|T u-T x_{2 n+1}, z\right\|,
\end{aligned}
$$

and if in the last inequality we take $n \rightarrow \infty$ we get that for each $z \in L$ the following holds true $\left\|T u-T S_{1} u, z\right\| \leq \alpha\left\|T u-T S_{1} u, z\right\|$, and how $\alpha<1$, from the last inequality follows $\left\|T S_{1} u-T u, z\right\|=0$, for each $z \in L$. Now, as in the proof of Theorem 1 we can conclude that $u$ is fixed point of $S_{1}$. Analogously can be proved that $u$ is fixed point of $S_{2}$. Let $v \in L$ is another fixed point of $S_{2}$, i.e. $S_{2} v=v$. For each $z \in L$ the following holds true

$$
\begin{aligned}
\|T u-T v, z\| & =\left\|T S_{1} u-T S_{2} v, z\right\| \\
& \leq \alpha\left(\left\|T u-T S_{2} v, z\right\|+\left\|T v-T S_{1} u, z\right\|\right)+\gamma\|T u-T v, z\| \\
& =(2 \alpha+\gamma)\|T u-T v, z\| .
\end{aligned}
$$

Since $2 \alpha+\gamma<1$ from the last inequality it follows that for every $z \in L$ the following holds true $\|T u-T v, z\|=0$, from which follows that $T u=T v$. But, $T$ is injection, so $u=v$.

Corollary 4. Let $(L,\|\cdot, \cdot\|)$ be a 2-Banach space, $S_{1}, S_{2}: L \rightarrow L$ and the mapping $T: L \rightarrow L$ is continuous, injection and sequentially convergent. If $\alpha>0, \gamma \geq 0$ are such that $2 \alpha+\gamma<1$ and

$$
\left\|T S_{1} x-T S_{2} y, z\right\| \leq \alpha \frac{\left\|T x-T S_{2} y, z\right\|^{2}+\left\|T y-T S_{1} x, z\right\|^{2}}{\left\|T x-T S_{2} y, z\right\|+\left\|T y-T S_{1} x, z\right\|}+\gamma\|T x-T y, z\|,
$$

for each $x, y, z \in L, z \neq 0$, then $S_{1}$ and $S_{2}$ have a unique common fixed point $u \in L$.

Proof. From inequality of condition follows inequality (4). Now the assertion follows from Theorem 2 .

Corollary 5. Let $(L,\|\cdot, \cdot\|)$ be a 2 -Banach space, $S_{1}, S_{2}: L \rightarrow L$ and mapping $T: L \rightarrow L$ is continuous, injection and sequentially convergent. If $0<\lambda<1$ and

$$
\left\|T S_{1} x-T S_{2} y, z\right\| \leq \lambda \cdot \sqrt[3]{\left\|T x-T S_{2} y, z\right\| \cdot\left\|T y-T S_{1} x, z\right\| \cdot\|T x-T y, z\|},
$$

for each $x, y, z \in L$, then $S_{1}$ and $S_{2}$ have a unique common fixed point $z \in L$. 
Proof. From the inequality between the arithmetic and geometric mean follows that

$$
d\left(T S_{1} x, T S_{2} y\right) \leq \frac{\lambda}{3}\left(d\left(T x, T S_{2} y\right)+d\left(T y, T S_{1} x\right)+d(T x, T y)\right) .
$$

Now the assertion follows from Theorem 2 for $\alpha=\gamma=\frac{\lambda}{3}$.

Corollary 6. Let $(L,\|\cdot, \cdot\|)$ be a 2-Banach space, $S_{1}^{p}, S_{2}^{q}: L \rightarrow L, p, q \in \mathbf{N}$ and mapping $T: L \rightarrow L$ is continuous, injection and sequentially convergent. If $\alpha>0, \gamma \geq 0$ are such that $2 \alpha+\gamma<1$ and

$$
\left\|T S_{1}^{p} x-T S_{2}^{q} y, z\right\| \leq \alpha\left(\left\|T x-T S_{2}^{q} y, z\right\|+\left\|T y-T S_{1}^{p} x, z\right\|\right)+\gamma\|T x-T y, z\|,
$$

for each $x, y, z \in L$. Then $S_{1}$ and $S_{2}$ have a unique common fixed point $u \in L$. Proof. The proof is identical to the proof of the corollary 5 .

Remark 2. The mapping $T: L \rightarrow L$ determined by $T x=x, x \in L$ is sequentially convergent. Therefore, if in Theorem 2 and corollaries 4,5 and 6 we take $T x=x$, follows the correctness of Theorem 5 and corollaries 9, 10 и 11, [7].

\section{COMMON FIXED POINTS OF MAPPINGS OF KOPARDE-WAGHMODE TYPE}

Theorem 3. Let $(L,\|\cdot, \cdot\|)$ be a 2-Banach space, $S_{1}, S_{2}: L \rightarrow L$ and mapping $T: L \rightarrow L$ is continuous, injection and sequentially convergent. If $\alpha>0, \gamma \geq 0$, $2 \alpha+\gamma<1$ and

$\left\|T S_{1} x-T S_{2} y, z\right\|^{2} \leq \alpha\left(\left\|T x-T S_{1} x, z\right\|^{2}+\left\|T y-T S_{2} y, z\right\|^{2}\right)+\gamma\|T x-T y, z\|^{2}$,

for each $x, y, z \in L$, then $S_{1}$ and $S_{2}$ have a unique common fixed point $u \in L$.

Proof. Let $x_{0}$ be an arbitrary point of $L$ and let the sequence $\left\{x_{n}\right\}$ is defined with $x_{2 n+1}=S_{1} x_{2 n}, x_{2 n+2}=S_{2} x_{2 n+1}$, for $n=0,1,2, \ldots$. If there is an $n \geq 0$ such that $x_{n}=x_{n+1}=x_{n+2}$, then $u=x_{n}$ is a common fixed point for $S_{1}$ and $S_{2}$. Therefore, let's assume that there do not exist three consecutive equal members of the sequence $\left\{x_{n}\right\}$. Then, from inequality (6) follows that for each $n \geq 1$ and for each $z \in L$ the following holds true

$$
\begin{aligned}
\left\|T x_{2 n+1}-T x_{2 n}, z\right\|^{2} \leq & \alpha\left(\left\|T x_{2 n}-T x_{2 n+1}, z\right\|^{2}+\left\|T x_{2 n-1}-T x_{2 n}, z\right\|^{2}\right) \\
& +\gamma\left\|T x_{2 n}-T x_{2 n-1}, z\right\|^{2}
\end{aligned}
$$

and 


$$
\begin{gathered}
\left\|T x_{2 n-1}-T x_{2 n}, z\right\|^{2} \leq \\
\alpha\left(\left\|T x_{2 n-2}-T x_{2 n-1}, z\right\|^{2}+\left\|T x_{2 n-1}-T x_{2 n}\right\|^{2}\right) \\
+\gamma\left\|T x_{2 n-2}-T x_{2 n-1}, z\right\|^{2}
\end{gathered}
$$

from which it follows that for each $n=0,1,2, \ldots$ and for each $z \in L$ the following holds true

$$
\left\|T x_{n+1}-T x_{n}, z\right\| \leq \lambda\left\|T x_{n}-T x_{n-1}, z\right\|,
$$

where $\lambda=\sqrt{\frac{\alpha+\gamma}{1-\alpha}}<1$. Now from inequality (7) follows

$$
\left\|T x_{n+1}-T x_{n}, z\right\| \leq \lambda^{n}\left\|T x_{1}-T x_{0}, z\right\|,
$$

for each $n=0,1,2, \ldots$ and for each $z \in L$. Furthermore, from inequality (8), in the same way as in the proof of Theorem 1 it follows that the sequence $\left\{T x_{n}\right\}$ is convergent, and therefore the sequence $\left\{x_{n}\right\}$ is convergent also, i.e. exists $u \in X$ such that $\lim _{n \rightarrow \infty} x_{n}=u$ and $\lim _{n \rightarrow \infty} T x_{n}=T u$. We will prove that $u$ is fixed point of $S_{1}$. We have

$$
\begin{aligned}
& \left\|T u-T S_{1} u, z\right\| \leq\left\|T u-T x_{2 n+2}, z\right\|+\left\|T x_{2 n+2}-T S_{1} u, z\right\| \\
& =\left\|T u-T x_{2 n+2}, z\right\|+\left\|T S_{1} u-T S_{2} x_{2 n+1}, z\right\| \\
& \leq\left\|T u-T x_{2 n+2}, z\right\|+\sqrt{\alpha\left(\left\|T u-T S_{1} u, z\right\|^{2}+\left\|T x_{2 n+1}-T S_{2} x_{2 n+1}, z\right\|^{2}\right)+\gamma\left\|T u-T x_{2 n+1}, z\right\|^{2}} \\
& =\left\|T u-T x_{2 n+2}, z\right\|+\sqrt{\alpha\left(\left\|T u-T S_{1} u, z\right\|^{2}+\left\|T x_{2 n+1}-T x_{2 n+2}, z\right\|^{2}+\gamma\left\|T u-T x_{2 n+1}, z\right\|^{2}\right.}
\end{aligned}
$$

for each $n \in \mathbf{N}$ and for each $z \in L$. If in the last inequality we take $n \rightarrow \infty$ we get that

$$
\left\|T u-T S_{1} u, z\right\| \leq \sqrt{\alpha} d\left\|T u-T S_{1} u, z\right\|,
$$

for each $z \in L$ and how $\sqrt{\alpha}<1$, it follows that $\left\|T u-T S_{1} u, z\right\|=0$. Now, again as in the proof of Theorem 1 we conclude that $u$ is fixed point of $S_{1}$. Analogously it can be proved that $u$ is fixed point of $S_{2}$. Let $v \in L$ be another fixed point of $S_{2}$, i.e. $S_{2} v=v$. Then, for each $z \in L$ the following holds true

$$
\begin{aligned}
\|T u-T v, z\|^{2} & =\left\|T S_{1} u-T S_{2} v, z\right\|^{2} \\
& \leq \alpha\left(\left\|T u-T S_{1} u, z\right\|^{2}+\left\|T v-T S_{2} v, z\right\|^{2}\right)+\gamma\|T u-T v, z\|^{2} \\
& =\gamma\|T u-T v, z\|^{2},
\end{aligned}
$$

and how $0 \leq \beta<1$ we get that $\|T u-T v, z\|=0$, from where it follows that $T u=T v$. But, $T$ is injection, so $u=v$. 
Corollary 7. Let $(L,\|\cdot \cdot\|)$ be a 2-Banach space, $S_{1}^{p}, S_{2}^{q}: L \rightarrow L, p, q \in \mathbf{N}$ and mapping $T: L \rightarrow L$ is continuous, injection and sequentially convergent. If $\alpha>0, \gamma \geq 0$ are such that $2 \alpha+\gamma<1$ and

$$
\left\|T S_{1}^{p} x-T S_{2}^{q} y, z\right\|^{2} \leq \alpha\left(\left\|T x-T S_{1}^{p} x, z\right\|^{2}+\left\|T y-T S_{2}^{q} y, z\right\|^{2}\right)+\gamma\|T x-T y, z\|^{2},
$$

for each $x, y, z \in L$. Then $S_{1}$ and $S_{2}$ have a unique common fixed point $u \in L$.

Proof. The proof is identical to the proof of the corollary 6 .

Remark 3. The mapping $T: L \rightarrow L$ determined by $T x=x, x \in L$ is sequentially convergent. Therefore, if in Theorem 3 and corollary 7 we take $T x=x$, it follows the correctness of Theorem 6 and corollary 12, [7].

\section{CONFLICT OF INTEREST}

No conflict of interest was declared by the authors.

\section{AUTHOR's CONTRIBUTIONS}

All authors contributed equally and significantly to writing this paper. All authors read and approved the final manuscript.

\section{References}

[1] A. Malčeski, R. Malčeski, A. Ibrahimi, Extending Kannan and Chatterjea Theorems in 2Banach Spaces by using Sequentialy Convergent Mappings, Matematichki bilten, Vol. 40, No. 1 (2016), 29-36

[2] A. Malčeski, S. Malčeski, K. Anevska, R. Malčeski, New Extension of Kannan and Chatterjea Fixed Point Theorems on Complete Metric Spaces, British Journal of Mathematics \& Computer Science, Vol. 17, No. 2, (2016), 1-10

[3] A. White, 2-Banach Spaces, Math. Nachr. Vol. 42 (1969), 43-60

[4] M. Kir, H. Kiziltunc, Some New Fixed Point Theorems in 2-Normed Spaces, Int. Journal of Math. Analysis, Vol. 7 No. 58 (2013), 2885-2890

[5] P. Chouhan, N. Malviya, Fixed Points of Expansive Type Mappings in 2Banach Spaces, International Juornal of Analysis and Applications, Vol. 3 No. 1 (2013), 60-67 
[6] P. K. Hatikrishnan, K. T. Ravindran, Some Properties of Accretive Operators in Linear 2-Normed Spaces, International Mathematical Forum, Vol. 6 No. 59 (2011), 2941-2847

[7] R. Malčeski, A. Ibrahimi, Contraction Mappings and Fixed Point in 2Banach Spaces, IJSIMR, Vol. 4, Issue 4, pp. 34-43

[8] R. Malčeski, K. Anevska, About the 2-Banach spaces, International Journal of Modern Engineering Research (IJMER), Vol. 4 Iss. 5 (2014), 28-32

[9] R. Malčeski, A. Malčeski, K. Anevska, S. Malčeski, Common Fixed Points of Kannan and Chatterjea Types of Mappings in a Complete Metric Space, British Journal of Mathematics \& Computer Science, Vol. 18, No. 1, (2016), 1-11

\footnotetext{
${ }^{1)}$ Faculty of informatics, University Goce Delchev, Shtip, Macedonia E-mail address: martin.lukarevski@ugd.edu.mk

${ }^{2)}$ Centre for research and development of education, Skopje, Macedonia E-mail address: samoil.malcheski@gmail.com
} 